Preliminary report for analysis of genome wide mutations from four ciprofloxacin resistant B. anthracis Sterne isolates generated by Illumina, 454 sequencing and microarrays for DHS

C. Jaing, L. Vergez, A. Hinckley, J. Thissen, S. Gardner, K. McLoughlin, P. Jackson

June 23, 2011 
This document was prepared as an account of work sponsored by an agency of the United States government. Neither the United States government nor Lawrence Livermore National Security, LLC, nor any of their employees makes any warranty, expressed or implied, or assumes any legal liability or responsibility for the accuracy, completeness, or usefulness of any information, apparatus, product, or process disclosed, or represents that its use would not infringe privately owned rights. Reference herein to any specific commercial product, process, or service by trade name, trademark, manufacturer, or otherwise does not necessarily constitute or imply its endorsement, recommendation, or favoring by the United States government or Lawrence Livermore National Security, LLC. The views and opinions of authors expressed herein do not necessarily state or reflect those of the United States government or Lawrence Livermore National Security, LLC, and shall not be used for advertising or product endorsement purposes.

This work performed under the auspices of the U.S. Department of Energy by Lawrence Livermore National Laboratory under Contract DE-AC52-07NA27344. 


\title{
Preliminary report for analysis of genome wide mutations from four ciprofloxacin resistant $B$. anthracis Sterne isolates generated by Illumina, 454 sequencing and microarrays for DHS
}

\author{
Contributors: \\ Crystal Jaing, Lisa Vergez, Aubree Hinckley, James Thissen, Shea Gardner, Kevin McLoughlin, \\ Paul Jackson \\ Lawrence Livermore National Laboratory (LLNL), Livermore, CA \\ Sally Ellingson, Loren Hauser and Tom Brettin \\ Computational Biology and Bioinformatics Group \\ Oak Ridge National Laboratory \\ Viacheslav Fofanov, Heather Koshinsky \\ Eureka Genomics, Hercules, CA \\ Yuriy Fofanov \\ University of Houston \\ Principal Investigator and Correspondent \\ Crystal Jaing \\ 925-424-6574, jaing2@1lnl.gov \\ Paul Jackson \\ (925) 424-2725, jackson80@1lnl.gov
}

This document was prepared as an account of work sponsored by an agency of the United States government. Neither the United States government nor Lawrence Livermore National Security, LLC, nor any of their employees makes any warranty, expressed or implied, or assumes any legal liability or responsibility for the accuracy, completeness, or usefulness of any information, apparatus, product, or process disclosed, or represents that its use would not infringe privately owned rights. Reference herein to any specific commercial product, process, or service by trade name, trademark, manufacturer, or otherwise does not necessarily constitute or imply its endorsement, recommendation, or favoring by the United States government or Lawrence Livermore National Security, LLC. The views and opinions of authors expressed herein do not necessarily state or reflect those of the United States government or Lawrence Livermore National Security, LLC, and shall not be used for advertising or product endorsement purposes.

This work performed under the auspices of the U.S. Department of Energy by Lawrence Livermore National Laboratory under Contract DE-AC52-07NA27344. 


\section{INTRODUCTION}

The objective of this project is to provide DHS a comprehensive evaluation of the current genomic technologies including genotyping, Taqman PCR, multiple locus variable tandem repeat analysis (MLVA), microarray and high-throughput DNA sequencing in the analysis of biothreat agents from complex environmental samples. As the result of a different DHS project, we have selected for and isolated a large number of ciprofloxacin resistant B. anthracis Sterne isolates. These isolates vary in the concentrations of ciprofloxacin that they can tolerate, suggesting multiple mutations in the samples. In collaboration with University of Houston, Eureka Genomics and Oak Ridge National Laboratory, we analyzed the ciprofloxacin resistant B. anthracis Sterne isolates by microarray hybridization, Illumina and Roche 454 sequencing to understand the error rates and sensitivity of the different methods. The report provides an assessment of the results and a complete set of all protocols used and all data generated along with information to interpret the protocols and data sets.

\section{METHODS}

\section{Bacillus anthracis Sterne ciprofloxacin selections}

A parental culture of avirulent Bacillus anthracis Sterne was streaked onto a nutrient broth agar plate. The wild-type ciprofloxacin minimum inhibitory concentration (MIC) value was determined for $B$. anthracis Sterne by picking a single colony to inoculate $5 \mathrm{~mL}$ nutrient broth and incubating overnight at $35^{\circ} \mathrm{C}, 160 \mathrm{rpm}$. A subculture containing $5 \mathrm{~mL}$ nutrient broth was inoculated with $200 \mu \mathrm{L}$ of the overnight culture and incubated at $35^{\circ} \mathrm{C}, 160 \mathrm{rpm}$ to an optical density at $600 \mathrm{~nm}$ of 0.8. A ciprofloxacin Etest (AB Biodisk) was applied to a nutrient broth agar plate swabbed for full coverage with the $B$. anthracis Sterne subculture, and the Etest plate was incubated overnight at $35^{\circ} \mathrm{C}$. An approximate ciprofloxacin MIC was determined to be $0.047 \mu \mathrm{g} / \mathrm{mL}$ for the wild-type B. anthracis Sterne.

Cultures were prepared for first-round selections by inoculating 20 tubes containing $5 \mathrm{~mL}$ nutrient broth, each with a single $B$. anthracis Sterne colony. The tubes were incubated horizontally at $35^{\circ} \mathrm{C}, 160 \mathrm{rpm}$, overnight. Fresh subcultures were prepared by adding $500 \mu \mathrm{L}$ of each overnight culture to $12 \mathrm{~mL}$ nutrient broth. The subcultures were incubated at $35^{\circ} \mathrm{C}, 160$ $\mathrm{rpm}$ to an optical density at $600 \mathrm{~nm}$ of 0.8 . The cells were concentrated by centrifugation at $4000 x \mathrm{~g}$ for $10 \mathrm{~min}$. Approximately $11.5 \mathrm{~mL}$ of the supernatant was discarded and each cell pellet was suspended in the remaining $1 \mathrm{~mL}$ nutrient broth. Each of the twenty $1 \mathrm{~mL}$ suspensions was plated on a nutrient broth agar plate containing $0.094 \mu \mathrm{g} / \mathrm{mL}$ ciprofloxacin (three times the wild-type MIC value). These 20 first-round selection plates were incubated at $35^{\circ} \mathrm{C}$, up to 72 hours. Each of the small number of ciprofloxacin resistant colonies was picked into $5 \mathrm{~mL}$ nutrient broth containing $0.094 \mu \mathrm{g} / \mathrm{mL}$ ciprofloxacin (three times the wild-type MIC value) and incubated at $35^{\circ} \mathrm{C}, 160 \mathrm{rpm}$ up to 72 hours. Subcultures were prepared from any passage cultures that grew in the presence of ciprofloxacin by adding $200 \mu \mathrm{L}$ of the passage culture to $5 \mathrm{~mL}$ nutrient broth without ciprofloxacin and incubating at $35^{\circ} \mathrm{C}, 160 \mathrm{rpm}$ to an optical density at $600 \mathrm{~nm}$ of 0.8 . These subcultures were used for MIC value determinations (as indicated above) and for frozen stocks by adding $775 \mu \mathrm{L}$ of the subculture to $225 \mu \mathrm{L}$ sterile $80 \%$ glycerol followed by storage at $-80^{\circ} \mathrm{C}$.

Second and third round selections were performed by repeating this process to obtain

mutants resistant to ciprofloxacin concentrations well above therapeutic levels. Second round 
selections of the first-round mutants were carried out by increasing ciprofloxacin concentrations to approximately three-fold the parent generation MIC values at each step. Approximately 10 second-round mutants were carried on for each of 20 first-round mutants, and up to 5 third-round mutants were saved for each successful second-round mutant.

Mutant colonies were verified to be $B$. anthracis Sterne by colony morphology and species-specific PCR. Heat soak lysates were used as PCR templates. Heat soaks were prepared by transferring a single mutant colony into $200 \mu \mathrm{L}$ filter-sterilized $1 \mathrm{X}$ TE and incubating at $95^{\circ} \mathrm{C}$ for 20 minutes. The sample was cooled to room temperature and centrifuged at 10,000 xg for 1 minute. The supernatant was transferred to a new tube and stored at $-20^{\circ} \mathrm{C}$. A summary of the selection process is shown in Figure 1.

\section{Avirulent Wild Type B. anthracis Sterne}

$0.12 \mu \mathrm{g} / \mathrm{ml}$ MIC Ciprofloxacin

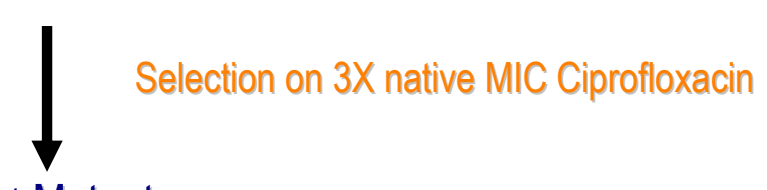

Step 1 Mutants

Three phenotypes $/ 2$ - $12 \mu \mathrm{g} / \mathrm{ml}$ MIC
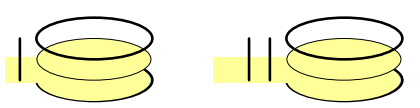

Selection on 3X Step 1 MIC Ciprofloxacin

Step 2 Mutants

Five phenotypes $/ 8$ - >32 $\mu \mathrm{g} / \mathrm{ml}$ MIC
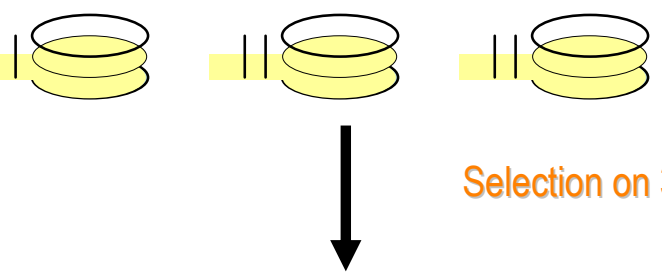

Selection on 3X Step 2 MIC Ciproflaxacin

Step 3 Mutants

Five phenotypes/24 - $100 \mu \mathrm{g} / \mathrm{ml}$ MIC
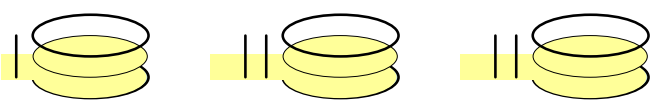

1

\section{Genomic DNA preparations, PCR and sequence verification}


Genomic DNA from different isolates was isolated using the Epicentre Masterpure Gram Positive DNA kit. PCR oligonucleotide primers that amplified genome-specific sequences from the different microbial species were designed using Primer3TM. PCR used Promega reagents. Sequence verification of a targeted PCR region, when required, was performed by primer walking off the PCR products using $1 / 8$ of a Big Dye V3.1 sequencing kit. Sanger ${ }^{\mathrm{TM}}$ sequencing was using ABI3730 DNA analyzers at the DOE Joint Genome Institute in Walnut Creek, CA.

\section{Illumina Sequencing}

\subsection{Illumina sequencing experimental procedure}

Genomic DNA samples from the Bacillus anthracis Sterne ciprofloxacin mutants were provided to Eureka Genomics for Illumina sequencing. The samples were sequenced according to standard Illumina sequencing procedures.

\subsection{Illumina sequence data generation and quality control}

Illumina paired end libraries were prepared from $1 \mu \mathrm{g}$ of genomic DNA (gDNA) from each of the eleven third round CIP resistant isolates, for the purpose of single-end sequencing on the Genome Analyzer IIx. Briefly, the gDNA was fragmented, end repaired, A' tagged, ligated to adaptors, size-selected and enriched with 13 cycles of PCR. Each library was assigned one lane of a flow cell to undergo cluster amplification and sequencing on the Genome Analyzer IIx, and 36 cycles of single-end sequence data was generated. The resulting sequencing reads were filtered using the default parameters of the Illumina QC pipeline (Bustard + Gerald).

As an additional quality control step, all reads were analyzed using the PIQA pipeline (16), which examines genomic reads produced by Illumina machines and provides tile-by-tile and cycle-by-cycle graphical representations of cluster density, quality scores, and nucleotide frequencies to allow easy identification of defective tiles, mistakes in sample/library preparations and abnormalities in the frequencies of appearance of sequenced genomic reads. All reads were determined to be of sufficient quality to proceed with subsequent analysis.

\subsection{Mapping and identifying candidate mutations}

The sequence reads from each of the samples were mapped with up to 1 mismatch to the reference $B$. anthracis Sterne genome (AE017225.1). To avoid uncertainty associated with identifying mutations in repeatable parts of the reference genome, for each position in the reference sequence a uniqueness score based on the subsequences covering this nucleotide was determined. Specifically, the copy number of each subsequence of size 36 (the length of reads used in sequencing) present in the reference genome was first calculated; the uniqueness score of each position in the reference genome was then defined as the total number of subsequences (factoring in the copy number) which covered this position. For example, in this metric, the score of 36 will appear only if each subsequence covering a given nucleotide is unique in the reference; higher scores indicate that one or more subsequences are present in the reference in several copies. $94.11 \%(1,784,242$ bases) of the reference genome has a uniqueness score of 36 . Mutations in these positions can be detected without the ambiguity caused by the presence of repeatable regions.

A given position is predicted to contain a mutation if: (1) the number of reads confirming the mutation on each strand exceeds the minimum count threshold-ensuring that only positions that achieve the minimum required coverage are considered, and (2) the proportion of reads confirming a mutation out of all the reads covering a given position exceeds a ratio thresholdensuring that only mutations that have the minimum required support are identified. As a 
compromise between mutation detection sensitivity and false discovery rate, the minimum count threshold was set at $10 \%$ of the median of the nucleotide-by-nucleotide coverage for each sample, and the ratio threshold was set at $30 \%$ of the total coverage on a per-nucleotide basis. In the present analysis, mutations confirmed on both strands (if the number of reads supporting the mutation exceeds the minimum count threshold on each of the strands separately) are distinguished from mutations for which such condition was met on only one strand. In the case of insertions, the mapping process results in the association of both perfect matches (PM) and insertions to the same location on the reference genome. Thus different ratio threshold criteria are used to detect different types of mutations at a given genome position. The criterion for detecting a substitution of base $B$ for the reference base is:

$$
\frac{S u b B^{+}+S u b B^{-}}{P M^{+}+P M^{-}+D e l^{+}+D e l^{-}+S u b A C T G^{+}+S u b A C T G^{-}} \geq \text {ratio threshold }
$$

The criterion for detecting a deletion is:

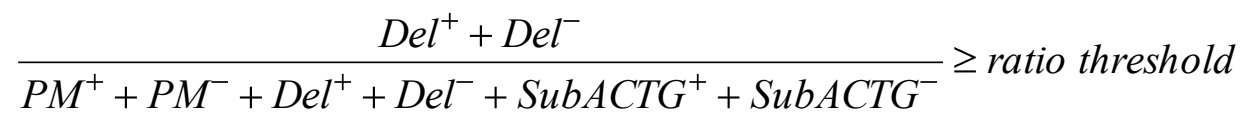

The criterion for detecting an insertion of base $B$ on the plus strand is:

$$
\frac{\operatorname{Ins} B^{+}+\operatorname{Ins} B^{-}}{\mathrm{Del}^{+}+\mathrm{Del}^{-}+\mathrm{SubACTG^{+ }}+\operatorname{SubACTG^{-}}+\operatorname{Ins} A C T G^{+}+\operatorname{Ins} A C T G^{-}} \geq \text {ratio threshold }
$$

In the numerators of the above formulas, $\mathrm{SubB}^{+/}, \mathrm{Del}^{+/}$, and $\mathrm{Ins} \mathrm{B}^{+/-}$stand for the numbers of reads confirming a substitution, deletion, or insertion, respectively, mapping to the genome strand indicated by the superscript. For substitutions and insertions, $S u b B^{-}$and Ins $^{-}$ indicate the numbers of reads mapped to the minus strand in which the base complementary to $B$ is substituted or inserted. In the denominators, the variables PM, SubACTG, and InsACTG respectively indicate the numbers of reads confirming a perfect match (PM), a substitution of any base, or an insertion of any base, at the genome position of interest.

While paired end data was generated, the reads were decoupled and a single-end read assembly (using in house algorithms) was performed on each of the sequence data sets. These contigs are shorter in length than contigs obtained with paired end data, but in general have fewer errors. Each mutation identified in each sample was confirmed to be present on the contigs assembled for that sample. Mutations (including insertions, deletions, and substitutions) that pass both thresholds and appear on both strands are less likely to be artifacts of sequencing read generation or artifacts of mapping. Mutations that only appear on one strand and cannot be verified on the opposite strand (something that is not common, given sufficient coverage), such as insertions, other than ' $G$ ' after ' $G$ ', ' $C$ ' after ' $C$ ', ' $A$ ' after ' $A$ ', and ' $T$ ' after ' $T$ ' are either artifacts of sequencing/mapping (false positives) or positions in the genome that did not have sufficient coverage to be verified on both strands.

\section{454 Sequencing Experimental Procedures. 4.1 454 sequencing procedures}

Genomic DNA samples from the Bacillus anthracis Sterne ciprofloxacin mutants were provided to the DNA Sequencing Center at Brigham Young University. The samples were 
sequenced according to standard Roche 454 procedures. Each mutant was sequenced per halfplate of the run with a total of 2454 Life sciences Titanium runs on the Genome Sequencer FLX. Bases on these parameters it was approximated that $\sim 166$ million bases of sequence data would be generated with a median read length of 242 bases.

\subsection{4 analysis procedures}

The 454 sequence reads were provided to Oak Ridge National Laboratory (ORNL) by LLNL. The quality filtered reads and quality scores were sent in FASTA and SFF formats. Reads were generated for the four ciprofloxacin resistant strains (M1-1, M1-6, M10-8-1, M19-2), as well as the non-resistant parental strain (Dugway).

Since the Sterne strain is the most recent fully annotated ancestor, reads from each of the mutant strains as well as the parental strain were mapped to the Sterne chromosome (GenBank: AE017225.1) and the annotated pX01 plasmid (GenBank: AF065404.1) using the mapping software (gsMapper) provided by the vender (Roche) of the sequencing instrument.

Reads were initially mapped using $100 \%, 97 \%, 94 \%, 91 \%$, and $90 \%$ minimum identity thresholds between the reads and reference to obtain an understanding of the data set. One hundred percent, $97 \%, 94 \%$, and $91 \%$ are approximately equivalent to $0,1,2$, and 3 mismatches in a 35 base pair read (thus these thresholds correspond to the thresholds available in Illumina mapping software used in the complementary experiment). Ninety percent was used in subsequent genomic variation analysis since it is the default recommended for the software and based on a survey of the different thresholds seemed to be reasonable. Mapping statistics for the mappings using 90\% minimum identity were parsed from the output and are presented.

Mapping the reads from one dataset (one mutant or the parental Dugway isolate of $B$. anthracis Sterne strain) to the $B$. anthracis Sterne genome would take $<10$ minutes at the highest stringency (100\% identity), but would take over an hour at the lowest levels (90\% identity). Therefore, once all the scripts were prepared to process the data, all the mappings could be done overnight. This would be followed by a combination of parsing some of the data using scripts and some of it manually.

For each mapping gsMapper provides a high quality difference file representing SNPs and indels. SNPs and indels for each strain were taken from the 'variants' tab in the gsMapper software. Variants from mapping the parental strain (Dugway) to Sterne were assumed to represent variations accumulated prior to antibiotic resistance selection or as potential sequencing errors in the reference genome or parental strain. Variants detected from each resistant strain that were not in the parental strain were found by selectively removing rows corresponding to SNPs in both the parental strain and in the resistant strain. The lowest quality score associated with each variation was obtained to identify detected variations that may be an error due to sequencing quality. All variants common among all the resistant strains were identified manually. In-house scripts were used to annotate (gene locus/protein id, etc.) the positions of all the SNPs and indels. There were two SNPs common in all four of the resistant strains that (a) were not present in the parental strain, and (b) were present in regions that encode proteins. The protein variants of these two SNPs were found manually.

Large deletions that are identified as unmapped portions of the reference genome were found using the mappings between the reads and Sterne reference. The 454alignmentinfo.tsv file generated by the gsMapper software was parsed to find large regions of the reference genome that had a consensus base of "-" in the mapping. These regions had a total depth of greater than or equal to 2, but no unique depth. Regions omitted from 454alignmentinfo.tsv were also found 
using the same script. These regions have no consensus base, i.e. regions with a total depth less than 2 , even if it had a unique depth of 1 . To discern between areas with no consensus base that have a unique depth of 1 and those that do not, the 454AllContigs.fna file, generated by the gsMapper software, was parsed to find regions not mapped.

The 454ReadStatus.txt file, generated by the gsMapper software, was parsed using an inhouse script to find the identification (ID) of the reads not mapped for all mappings using $90 \%$ minimum identity.

\section{Microarray Experimental Procedures}

\subsection{Whole genome tiling array design for $B$. anthracis Sterne}

Tiling arrays were developed using the NimbleGen $388 \mathrm{~K}$ probe format for B. anthracis Sterne. We have developed computational tools to design probes that tile across entire bacterial genomes. Probes were isothermal to the extent possible within the allowed length range of 32-40 nucleotides. The $\mathrm{T}_{\mathrm{m}}$ ranges were selected to account for the $\mathrm{GC} \%$ of each organism to center the distribution of probe length around 36 nucleotides. The $\mathrm{T}_{\mathrm{m}}$ was calculated using Unafold (http://dinamelt.bioinfo.rpi.edu/download.php), which employs accurate nearest neighbor thermodynamic predictions.

B. anthracis Sterne probes were tiled with an overlap of $55 \%$ and $\mathrm{T}_{\mathrm{m}} 75 \pm 3^{\circ} \mathrm{C}$ across the sequences of gi|47566322|ref|NC_007322.2| Bacillus anthracis str. primeAmes Ancestorprime plasmid pXO1 gi|50163691|ref|NC_007323.3| Bacillus anthracis str. primeAmes Ancestorprime plasmid pXO2 gi|10956390|ref|NC_002146.1| Bacillus anthracis plasmid pX02 gi|10956247|ref|NC_001496.1| Bacillus anthracis plasmid pX01 ref|NC_005945.1|gnl|NCBI_GENOMES|405|gi|49183039|Bacillus anthracis str. Sterne Total \# probes: 370,803 with replicate on every $26^{\text {th }}$ position.

\subsection{Sample preparation and quantitation.}

All genomic DNA samples were sonicated prior to labeling and hybridization. Sonication was performed using a Branson Digital Sonifier S450D (Branson Ultrasonics, Danbury, CT) at a setting of $100 \%$ amplitude for 30 seconds. Sonication was repeated 4 times. The fragment sizes ranged from 500-2000 base pairs. Sample DNA concentrations were measured using a Nanodrop 1000 spectrophotometer (Thermo Scientific, Wilmington, DE).

Cy3-labeled random 9mers (TriLink Biotechnologies, San Diego, CA) were diluted using $125 \mathrm{mM}$ Tris- $\mathrm{HCl}, 12.5 \mathrm{mM} \mathrm{MgCl} 2,1.75 \%$ (v/v) b-mercaptoethanol at a concentration of 1 OD/42 $\mu \mathrm{L}$ of buffer. Approximately $1 \mu \mathrm{g}$ sonicated DNA samples in $40 \mu \mathrm{L}$ were mixed with an equal volume of $\mathrm{Cy} 3$-labeled random nonamer primers and the mixture was heat-denatured at $98^{\circ} \mathrm{C}$ for 10 minutes, then quick chilled on ice for 10 minutes. Ten $\mu \mathrm{L}$ of a solution containing $10 \mathrm{mM}$ dNTPs, $8 \mu \mathrm{L}$ PCR-grade water and $2 \mu \mathrm{L}$ of $50 \mathrm{U} / \mu \mathrm{L}$ Klenow DNA polymerase (New England Biolabs, Boston, MA) were added to the labeling reaction in a total volume of $100 \mu \mathrm{L}$ and the sample was incubated at $37^{\circ} \mathrm{C}$ for $2.5 \mathrm{hrs}$. The labeling reaction was stopped by addition of $10 \mu \mathrm{L} 0.5 \mathrm{M}$ EDTA. Labeled DNA was precipitated by addition of $11 \mu \mathrm{L} 3 \mathrm{M} \mathrm{NaCl}$ and 110 $\mu \mathrm{L}$ isopropanol, collected by centrifugation and the pellet was washed twice in $500 \mu \mathrm{L}$ of $80 \%$ $(\mathrm{v} / \mathrm{v})$ ethanol, and then dried. Once ready for hybridization, the pellet was dissolved in 10-20 $\mu \mathrm{L}$ PCR-grade water and the DNA concentration was again determined by $\mathrm{A}_{260}$ measurement using 
a Nanodrop spectrophotometer. Approximately $20 \mu \mathrm{g}$ of labeled DNA can be obtained from $\sim 1$ $\mu \mathrm{g}$ of starting genomic DNA.

A NimbleGen ${ }^{\mathrm{TM}}$ hybridization kit was purchased from Roche NimbleGen (Madison, WI). The kit contains hybridization buffers and alignment oligomers. For each hybridization reaction, $4 \mu \mathrm{g}$ of labeled DNA in $3.5 \mu \mathrm{L}$ was mixed with $31.5 \mu \mathrm{L} 2 \mathrm{x}$ Hybridization buffer, $8 \mu \mathrm{L}$ Hybridization component $\mathrm{A}$ and $1 \mu \mathrm{L}$ alignment oligomer (100 nM Cy3 and Cy5-labeled CPK6) in a total volume of $44 \mu \mathrm{L}$. The contents were mixed and denatured at $95^{\circ} \mathrm{C}$ for 5 minutes. The tube was immediately transferred to the MAUI 4-Bay ${ }^{\mathrm{TM}}$ Hybridization System (BioMicro Systems, Salt Lake City, UT) at $42^{\circ} \mathrm{C}$ until ready for sample loading. The MAUI Mixer SL Hybridization Chamber was placed on the array and the sample was loaded. The mixer was sealed and placed in the hybridization stations. Mix mode B was used and the samples were hybridized for 16-17 hrs. NimbleGen ${ }^{\mathrm{TM}}$ Wash Buffer kit was purchased from Roche NimbleGen. Each array was washed three times: Wash I, $42^{\circ} \mathrm{C}$ for $2 \mathrm{~min} 15 \mathrm{sec}$, Wash II, room temperature for $1 \mathrm{~min}$ and Wash III, room temperature for $15 \mathrm{sec}$. The arrays were removed from Wash III solution and spin dried in a slide-spinner (Labnet, Edison, $\mathrm{NJ}$ ) for $1 \mathrm{~min}$.

Arrays were scanned using an Axon 4000B scanner (Molecular Devices, Sunnyvale, CA) at $5 \mu \mathrm{m}$ resolution. A wavelength of $532 \mathrm{~nm}$ was used to scan for $\mathrm{Cy} 3$ dyes hybridized on microarray slides. The images of arrays were saved as a tiff file. NimbleScan software 2.4 was used to extract the array data from .tiff images and convert them into pair file reports. The locations of the alignment oligomers (CPK6 control probes) were used to lay the grid on the array. The pair reports were used for statistical analysis of microarray data.

\subsection{Statistical analysis of sequence changes from tiling microarrays.}

An algorithm called TAPS (Tiling Array Polymorphism Sensor) to analyze data from two-color hybridizations was developed. The TAPS algorithm is based on a thermodynamic model that predicts the effect of mutations on probe-target hybridization and estimates the likelihood of a mutation at every reference genome position, given the intensities of all probes overlapping the position,. The algorithm superficially resembles the SNPscanner algorithm of Gresham et al (3), but requires fewer training parameters (70 vs. 4608), and is thus less susceptible to over fitting; it also works with two-color data, and is not restricted to Affymetrix array designs.

The TAPS algorithm models the effect of a SNP on the intensity of an overlapping probe as a function of several variables: the reference channel probe intensity, the position of the SNP in the probe sequence, the base substitution relative to the reference genome, and the two perfect-match bases on either side of the SNP locus. We assume that probe intensity decreases as the free energy of hybridization increases (becomes less negative), and that the free energy $\Delta \mathrm{G}$ is a sum of contributions from aligned pairs of nearest-neighbor $(\mathrm{NN})$ nucleotides. A SNP in the target sequence increases the free energy by replacing two perfect-match NN pairs with pairs having a single mismatch. For example, a mutation that changes the sequence AGC to ATC replaces the perfect match pairs AG/TC and GC/CG with the mismatch pairs AT/TC and TC/CG. Since our tiling array only has probes for the reference genome sequence, it does not provide information about the specific base substitution in the target genome. However, the average effect of the 3 possible substitutions can be estimated within a particular base triplet. 
The TAPS model also includes a multiplicative position effect, in which SNPs near the middle of a probe cause larger intensity drops than SNPs hitting the ends, especially the 3' region closest to the array surface. We expected to see this based on our earlier work with virulence gene arrays (4). We see that, on average, the intensity drop is almost two-fold when a SNP affects the nucleotides binding near the middle of the probe, but is reduced to zero at either end.

Even in the absence of SNP effects, probe intensities will differ between the two channels due to dye effects, scanner bias and noise. To correct for these effects, each pair of intensities $\left(y_{\text {ref }}, y_{\text {mut }}\right)$ were transformed into the log ratio and log geometric mean:

$$
\begin{aligned}
& M=\log \frac{y_{\text {mut }}}{y_{\text {ref }}} \\
& A=\frac{1}{2}\left(\log y_{r e f}+\log y_{m u t}\right)
\end{aligned}
$$

A semi-parametric regression model was fit using the $M$ vs. $A$ data for all probes:

$$
M=\mu(A)+\varepsilon(A)
$$

in which the error term $\varepsilon(A)$ has mean 0 and variance $\sigma^{2}(A)$, and $\mu(A)$ and $\sigma^{2}(A)$ are smooth mean and variance functions. The functions $\mu(A)$ and $\sigma^{2}(A)$ to the $M$ and $A$ values were fit for all probes on each array, using regression on cubic splines to fit $\mu(A)$, and a smoothing spline on binned squared residuals to fit $\sigma^{2}(A)$. Since SNPs only affect a small fraction of probes on the array, the fitted $\mu(A)$ closely approximates the mean function for perfect match probes (those not overlapping variations between the reference and target strains).

To model the effect of a free energy change $\Delta \Delta G=\Delta G_{m u t}-\Delta G_{r e f}$ on the log intensity ratio, we assume that the probe oligos within an array feature can be in one of three states: unbound, bound to target DNA from the mutant strain, or bound to target DNA from the reference. At thermodynamic equilibrium at temperature $T$, the fraction of oligos bound to mutant DNA is given by the Boltzmann equation:

$$
\theta_{m u t}=\frac{e^{-\Delta G_{m u t} / R T}}{1+e^{-\Delta G_{r e f} / R T}+e^{-\Delta G_{m u t} / R T}}
$$

with a similar equation for the fraction bound to reference DNA $\theta_{\text {ref. }}$ It follows that

$$
\log \frac{\theta_{m u t}}{\theta_{\text {ref }}}=-\frac{\Delta \Delta G}{R T}
$$

Since the probe intensity for each dye (at levels well above background and below saturation) scales with the fraction of oligos bound to target labeled with that dye, we expect the SNP effect on the log intensity ratio to be proportional to $\Delta \Delta G$. That is, for probes overlapping SNPs, our semi parametric regression model was modified to include a term for the SNP effect:

$$
M_{o b s}=\mu\left(A_{o b s}\right)+w \Delta \Delta G+\varepsilon\left(A_{o b s}\right)
$$

where $w$ is a proportionality constant (typically $<0)$ and the noise term $\varepsilon(A)$ is assumed to be Gaussian with mean 0 and the same variance $\sigma^{2}(A)$ as was estimated for perfect match probes. The free energy effect $w \Delta \Delta G$ is modeled as a product of triplet and position effects:

$$
w \Delta \Delta G=\beta_{\tau} h(x)
$$

where $\tau$ indexes the triplet and $x$ is the position of the SNP within the probe, as a fraction of the probe length. The position effect $h(x)$ is approximated by a polynomial function of degree 5:

$$
h(x)=\sum_{j=0}^{5} \alpha_{j} x^{j}
$$


The triplet effects are assumed to be equivalent for reverse complements, so there are 32 $\beta_{\tau}$ parameters and $6 \alpha_{j}$ 's. Note that the proportionality constant $w$ has been absorbed into the triplet effects. The model parameters were fit to data from experiments in which the target (mutant) strain has known genome sequence and thus has SNPs at known positions relative to the reference genome. To make the parameters identifiable, we scaled the coefficients $\alpha_{j}$ so that $h(0.5)=1$.

To apply the model to data from target strains of unknown sequence, a log likelihood ratio test statistic for every position $z$ in the reference genome was computed. Let $P(z)$ be the set of probes overlapping position $z$, and let $M_{i}$ and $A_{i}$ be the log intensity ratio and average for probe $i$. The semi parametric regression model given above leads to the following expression for the log likelihood:

$$
\log L(z)=-\sum_{i \in P(z)} \frac{\left(M_{i}-\mu\left(A_{i}\right)-w \Delta \Delta G_{i}\right)^{2}}{2 \sigma^{2}\left(A_{i}\right)}
$$

If there is a SNP at position $z$, then $\Delta \Delta G_{i}$ for each probes was computed using the fitted model parameters; otherwise $\Delta \Delta G_{i}=0$ for all probes in $P(z)$. The log likelihood ratio is the difference of the $\log L(z)$ values computed under these two assumptions. Candidate SNP positions were identified by looking for regions of the genome where the log likelihood ratio exceeds a fixed threshold. Typically the threshold was set to 20; in tests with targets of known sequence, this threshold provided an optimal balance between false positive and false negative SNP predictions.

\section{RESULTS}

\section{Avirulent $B$. anthracis Sterne ciprofloxacin resistant isolates}

Following two rounds of selection by exposure to increasing ciprofloxacin concentrations, 3 ciprofloxacin resistant avirulent $B$. anthracis Sterne isolates were collected for this study. One mutant (10:8:1) was collected after three rounds of selection. The MIC value for the beginning sensitive Sterne strain was $0.047 \mu \mathrm{g} / \mathrm{mL}$. MIC values for the 4 resistant isolates ranged from $24 \mu \mathrm{g} / \mathrm{mL}$ to $>32 \mu \mathrm{g} / \mathrm{mL}$ ciprofloxacin (the limit of the Etest). It is not yet known whether this is a relationship between association with a particular MIC value and particular genomic changes responsible for resistance. Table 1 contains a compilation of the different resistant isolates and their MIC values.

Table 1. Avirulent B. anthracis Sterne - Ciprofloxacin resistant isolate MIC summary. All MIC values are in $\mu \mathrm{g} / \mathrm{ml}$.

\begin{tabular}{|c|c|c|c|c|c|}
\hline \multicolumn{6}{|c|}{ Avirulent $B$. anthracis Sterne wild-type Ciprofloxacin MIC (Etest) $=0.047 \mu \mathrm{g} / \mathrm{ml}$} \\
\hline \multicolumn{2}{|c|}{ Round 1 } & \multicolumn{2}{|c|}{ Round 2} & \multicolumn{2}{|c|}{ Round 3} \\
\hline Name & MIC & Name & MIC & Name & MIC \\
\hline M1 & 0.75 & M1:1 & 24.0 & M10:8:1 & $>32$ \\
\hline M10 & 1.0 & M1:6 & $>32$ & & \\
\hline M19 & 1.0 & M10:8 & 12.0 & & \\
\hline & & M19:2 & $>32$ & & \\
\hline
\end{tabular}

\section{Evaluation of resistant isolates using microarrays and sequencing technologies}

SNPs identified in ciprofloxacin-resistant avirulent B. anthracis Sterne isolates. We first tested the $B$. anthracis Sterne tiling microarray on reference (non-selected) B. anthracis Sterne. We then tested four ciprofloxacin resistant $B$. anthracis Sterne isolates (1:1, 1:6, 10:8:1, and 
19:2) using the $B$. anthracis Sterne tiling array. The number of candidate SNP's on the $B$. anthracis Sterne chromosome that were identified from overlapping probes was: 2078 in clone $1: 1,42$ in 1:6, 86 in clone 10:8:1, and 19 in 19:2. In addition, a $93 \mathrm{~kb}$ deletion relative to the reference strain was identified in the 10:8:1 isolate. The missing region spans positions 749405 to 842475 on the Sterne chromosome. Two of the overlapping high-scoring probe pairs are located in genes encoding the proteins targeted by ciprofloxacin, DNA gyrase A and topoisomerase IV. Several other SNP's are located in ABC transporter/permease genes while many others were in genes encoding hypothetical or un-annotated proteins.

The four ciprofloxacin-resistant B. anthracis Sterne isolates and the B. anthracis Sterne reference isolate were sent to Eureka Genomics to perform Illumina sequencing and BYU to perform 454 sequencing in order to compare the accuracy and cost-effectiveness of these two technologies for SNP detection, and to provide independent confirmation of the microarray results. Table 2 below displays the total number of SNPs identified by each method. Only those SNPs above a threshold of 0.30 for the sequencing technologies and those identified by 2 or more probes for the microarrays are included in this total number.

Table 2. Total SNPs identified by each technology

\begin{tabular}{|c|c|c|c|}
\hline $\begin{array}{c}\text { B. anthracis } \\
\text { Sterne } \\
\text { ciprofloxacin- } \\
\text { resistant clones }\end{array}$ & $\begin{array}{c}\text { \# of SNP's } \\
\text { identified by } \\
\text { microarray }\end{array}$ & $\begin{array}{c}\text { \# of SNP's } \\
\text { identified by } \\
\text { Illumina }\end{array}$ & $\begin{array}{c}\text { \# of SNP's } \\
\text { identified by } \\
\mathbf{4 5 4}\end{array}$ \\
\hline $1: 1$ & 2078 & 3 & 14 \\
\hline $1: 6$ & 42 & 207 & 8 \\
\hline $10: 8: 1$ & 86 & 13 & 10 \\
\hline $19: 2$ & 19 & 4 & 19 \\
\hline
\end{tabular}

In the following tables (2-5) the SNPs are detailed for each mutant. SNPs present in intergenic regions and very large deletions are not included here. For the sequencing technologies, the number of reads conferring each SNP is listed along with the proportion this represents. An (X) is marked in the Microarray column if at least two probes identified the SNP in the regions that concurred with the sequencing results. The only mutant with microarray data that matches with the sequencing technologies was 10:8:1 as shown below. B. anthracis Sterne tiling array did not product robust data that correlated with sequencing results. The probe design for $B$. anthracis Sterne used an overlap of $55 \%$ in order to fit all probes onto a $388 \mathrm{~K}$ array. The assay showed that this overlap is not sufficient for SNP identification using the tiling array. We have since developed a tiling array for $F$. tularensis LVS genome with $85 \%$ overlap of probes. Tiling array data showed that the consistency rate between microarray and sequencing is more than $95 \%$ (Jaing et al., manuscript in preparation). 
Table 3: Mutant 1:1 Sequencing Results Comparison

\begin{tabular}{|c|c|c|c|c|c|c|}
\hline Gene & Gene Description & Gene Location & $\begin{array}{c}\text { SNP } \\
\text { Location } \\
\end{array}$ & SNP Type & Illumina & 454 \\
\hline BAS0006 & DNA gyrase subunit A & $6596-9067$ & 6849 & Sub C->T & $20(1.00)$ & $19(1.00)$ \\
\hline BAS0627 & $\begin{array}{c}\mathrm{ABC} \text { transporter, nucleotide binding } \\
\text { domain }\end{array}$ & $677157-678104$ & 677934 & Del A & & $7(0.50)$ \\
\hline BAS0794 & $\begin{array}{l}\text { transcriptional regulator, TetR } \\
\text { family }\end{array}$ & $842297-842875$ & 842404 & Ins GC & & $17(1.00)$ \\
\hline BAS3009 & $\begin{array}{l}\text { penicillin-binding protein, } \mathrm{C} \text { - } \\
\text { terminus }\end{array}$ & 2983668-2984141 & 2983918 & Ins C & & $10(1.00)$ \\
\hline BAS3391 & DNA topoisomerase IV subunit A & $3363272-3365695$ & 3365454 & Sub G->T & $14(1.00)$ & $10(1.00)$ \\
\hline BAS3658 & $\begin{array}{l}\text { polyribonucleotide } \\
\text { nucleotidyltransferase }\end{array}$ & 3620153-3622291 & 3621030 & Ins AG & & $10(1.00)$ \\
\hline BAS3720 & $\begin{array}{l}\text { phosphopantothenoylcysteine } \\
\text { decarboxylase/phosphopantothenate- } \\
\text {-cysteine ligase }\end{array}$ & $3687114-3688319$ & $3687446-8$ & TCT->C & & $4(0.40)$ \\
\hline BAS5177 & $\begin{array}{l}\text { modification methylase, HemK } \\
\text { family }\end{array}$ & $5056920-5057173$ & 5057173 & Ins GAA & & $18(1.00)$ \\
\hline
\end{tabular}

Table 4: Mutant 1:6 Sequencing Results Comparison

\begin{tabular}{|c|c|c|c|c|c|c|}
\hline Gene & Gene Description & Gene Location & SNP Location & SNP Type & Illumina & 454 \\
\hline BAS0006 & DNA gyrase subunit A & $6596-9067$ & 6849 & Sub C->T & $8(1.00)$ & $11(1.00)$ \\
\hline \multirow{3}{*}{ BAS0425 } & \multirow{3}{*}{ hypothetical protein } & \multirow{3}{*}{$454916-455719$} & 455350 & Sub T->C & $11(0.92)$ & \\
\hline & & & 455353 & Sub T->A & $13(0.93)$ & \\
\hline & & & 455365 & Sub C->T & $9(1.00)$ & \\
\hline BAS0595 & sensory box/GGDEF family protein & $642479-644182$ & 643376 & Ins CCGCG & & $12(0.86)$ \\
\hline BAS0627 & $\begin{array}{c}\text { ABC transporter, nucleotide binding } \\
\text { domain }\end{array}$ & $677157-678104$ & 677934 & Del A & & $8(0.50)$ \\
\hline BAS0794 & transcriptional regulator, TetR family & $842297-842875$ & 842614 & $\begin{array}{l}\text { Ins } \\
\text { GCGGGTCTTGC }\end{array}$ & & $15(0.94)$ \\
\hline \multirow{2}{*}{ BAS3391 } & \multirow{2}{*}{ DNA topoisomerase IV subunit A } & \multirow{2}{*}{$3363272-3365695$} & \multirow{2}{*}{3365454} & Sub G->A & $8(0.89)$ & $12(0.86)$ \\
\hline & & & & Sub G->T & $1(0.11)$ & \\
\hline BAS5135 & $\begin{array}{l}\text { ABC transporter ATP-binding } \\
\text { protein, } \mathrm{N} \text {-terminus }\end{array}$ & $5019337-5019846$ & $5019628-30$ & ACA->C & & $4(0.33)$ \\
\hline BAS5220 & multidrug resistance protein, putative & $5109644-5108481$ & 5109171 & Sub C->A & $15(0.94)$ & \\
\hline
\end{tabular}


Table 5: Mutant 10:8:1 Sequencing and Microarray Results Comparison

\begin{tabular}{|c|c|c|c|c|c|c|c|}
\hline Gene & Gene Description & Gene Location & SNP Location & SNP Type & Illumina & 454 & $\begin{array}{l}\text { Micro- } \\
\text { array }\end{array}$ \\
\hline BAS0006 & DNA gyrase subunit A & $6596-9067$ & 6849 & Sub C->T & $22(1.00)$ & $11(1.00)$ & $\mathrm{X}$ \\
\hline \multirow{2}{*}{ tRNA-Leu-3 } & \multirow{2}{*}{ tRNA-Leu-3 } & \multirow{2}{*}{$746531-746614$} & 746559 & Sub T->C & $21(1.00)$ & & $\mathrm{X}$ \\
\hline & & & 746573 & Sub A->G & $21(1.00)$ & & $\mathrm{X}$ \\
\hline \multirow{4}{*}{ BAS0699 } & \multirow{4}{*}{$\begin{array}{l}\text { thiamine/molybdopterin biosynthesis } \\
\text { ThiF/MoeB-like protein }\end{array}$} & \multirow{4}{*}{$758236-759255$} & 758395 & Sub G->A & $10(1.00)$ & & $\mathrm{X}$ \\
\hline & & & 758412 & Sub C->T & $16(0.57)$ & & $\mathrm{X}$ \\
\hline & & & 758415 & Sub T->A & $16(0.57)$ & & $\mathrm{X}$ \\
\hline & & & 758436 & Sub G->A & $9(1.00)$ & & $\mathrm{X}$ \\
\hline \multirow{13}{*}{ BAS0757 } & \multirow{13}{*}{ hypothetical protein } & \multirow{13}{*}{$811432-812592$} & 812171 & Sub T->A & $19(1.00)$ & & $X$ \\
\hline & & & 812197 & Sub T->C & $22(1.00)$ & & $X$ \\
\hline & & & 812296 & Sub T->C & $32(1.00)$ & & $X$ \\
\hline & & & 812299 & Sub A->T & $36(1.00)$ & & $\mathrm{X}$ \\
\hline & & & 812300 & Sub C->T & $36(0.97)$ & & $\mathrm{X}$ \\
\hline & & & 812338 & Sub $A->G$ & $12(1.00)$ & & $X$ \\
\hline & & & 812339 & Sub G->A & $12(1.00)$ & & $\mathrm{X}$ \\
\hline & & & 812455 & Sub $\mathrm{T}->\mathrm{G}$ & $15(0.94)$ & & $\mathrm{X}$ \\
\hline & & & 812476 & Sub T->A & $46(1.00)$ & & $\mathrm{X}$ \\
\hline & & & 812485 & Sub T->G & $39(0.98)$ & & $\mathrm{X}$ \\
\hline & & & 812524 & Sub T->A & $10(1.00)$ & & $\mathrm{X}$ \\
\hline & & & 812535 & Sub T->A & $21(1.00)$ & & $X$ \\
\hline & & & 812560 & Sub C->T & $23(1.00)$ & & $\mathrm{X}$ \\
\hline \multirow{4}{*}{ BAS0786 } & \multirow{4}{*}{ hypothetical protein } & \multirow{4}{*}{$837472-837876$} & 837615 & Sub T->C & $15(1.00)$ & & $X$ \\
\hline & & & 837618 & Sub C->T & $17(1.00)$ & & $\mathrm{X}$ \\
\hline & & & 837642 & Sub T->C & $19(1.00)$ & & $X$ \\
\hline & & & 837646 & Sub A->C & $11(1.00)$ & & $X$ \\
\hline BAS1107 & $\begin{array}{l}\text { oligopeptide } \mathrm{ABC} \text { transporter, } \\
\text { oligopeptide-binding protein }\end{array}$ & $1161085-1162689$ & $1162599-600$ & Del AT & & $17(1.00)$ & \\
\hline BAS3391 & DNA topoisomerase IV subunit A & $3363272-3365695$ & 3365454 & Sub G->A & $33(1.00)$ & $9(1.00)$ & $\mathrm{X}$ \\
\hline BAS4080 & hemolysin A & $4014548-4015387$ & 4015055 & Ins ATC & & $14(1.00)$ & \\
\hline BAS4814 & hypothetical protein & $4706252-4707910$ & 4706846 & Sub T->C & $27(1.00)$ & $9(1.00)$ & \\
\hline BAS4948 & lipoprotein, putative & $4829169-4830134$ & 4829181 & Del A & & $8(0.89)$ & \\
\hline BAS5185 & stage 0 sporulation protein $\mathrm{F}$ & $5065837-5066205$ & $5065959-60$ & Del AA & & $13(1.00)$ & \\
\hline
\end{tabular}


Table 6: Mutant 19:2 Sequencing Results Comparison

\begin{tabular}{|c|c|c|c|c|c|c|}
\hline Gene & Gene Description & Gene Location & SNP Location & SNP Type & Illumina & 454 \\
\hline BAS0006 & DNA gyrase subunit A & $6596-9067$ & 6849 & Sub C->T & $45(1.00)$ & $12(1.00)$ \\
\hline BAS0276 & $\begin{array}{c}\text { phosphoribosylaminoimidazole carboxylase, } \\
\text { ATPase subunit }\end{array}$ & $296037-297188$ & 296553 & Sub C->T & & $17(1.00)$ \\
\hline BAS0361 & DNA topoisomerase III & $391511-393700$ & 391751 & Sub G->A & & $13(0.68)$ \\
\hline BAS0794 & transcriptional regulator, TetR family & $842297-842875$ & 842399 & Ins GC & & $22(1.00)$ \\
\hline BAS2000 & conserved domain protein & $2006552-2007553$ & 2006853 & Ins $\mathrm{T}$ & & $6(0.86)$ \\
\hline BAS3391 & DNA topoisomerase IV subunit A & $3363272-3365695$ & 3365454 & Sub G->A & $47(1.00)$ & $16(1.00)$ \\
\hline BAS3618 & DNA mismatch repair protein MutS & 3577813-3580491 & 3579082 & Ins GC & & $10(0.91)$ \\
\hline \multirow{2}{*}{ BAS3684 } & \multirow{2}{*}{ DNA topoisomerase I } & \multirow{2}{*}{ 3651076-3648998 } & 3651011 & Sub C->T & $44(1.00)$ & \\
\hline & & & 3651013 & Del C & & $6(1.00)$ \\
\hline BAS4278 & peptidase, U32 family & $4188897-4189826$ & 4188979 & Sub T->A & & $13(0.93)$ \\
\hline \multirow{7}{*}{ BAS4585 } & \multirow{7}{*}{ FtsK/SpoIIIE family protein } & \multirow{7}{*}{ 4485133-4489068 } & 4486921 & Sub T->C & & $3(0.60)$ \\
\hline & & & 4486948 & Sub T->C & & $6(0.86)$ \\
\hline & & & 4486987 & Sub T->C & & $8(1.00)$ \\
\hline & & & 4486990 & Ins TT & & $8(1.00)$ \\
\hline & & & 4486992 & Ins A & & $8(1.00)$ \\
\hline & & & 4487009 & Sub T->G & & $8(1.00)$ \\
\hline & & & 4487014 & Sub C->T & & $4(0.50)$ \\
\hline
\end{tabular}

In addition to those mutations detailed above, 454 sequencing also identified a $51 \mathrm{bp}$ deletion present in all 4 resistant mutants. The deletion sequence:

TAAATATGCCATGAATTATTTAACTGTTATATGAACCAAATAAAAAAAGCATTGCACAAGAGCA ATGCTTTTTTTATATATCCCGATCCAAATAAAGAGGTTA was located in an intergenic region from bases 3930400- 3930451.

A large deletion, 100722 bp long, located from positions 741,694 to 842,468 was identified in mutant 10:8:1. This deletion was identified by both sequencing technologies and the microarray analysis. 